\title{
Konzeptausbau und Beitragsentwicklung der Zeitschrift ONKOLOGIE VOn 1978-2002
}

\author{
W. Queißer
}

\section{Gründung und Aufbau}

In einem Redaktionsbüro in Starnberg entstand 1977 mit der Zeitschrift ONKOLOGIE eines der wichtigsten deutschsprachigen onkologischen Organe. Eine wichtige Rolle bei der Gründung der OnKologie spielte Prof. Dr. Dr. H. Wbra, der damalige Leiter des Instituts für Krebsforschung an der Universität Wien und Hauptschriftleiter der im S. Karger Verlag erscheinenden Oncology. Bei der Gründung wurde jedoch insbesondere darauf geachtet, der neuen Zeitschrift ein eigenes, von dem der Oncology klar abgegrenztes Profil zu geben. In den Jahren 1976 und 1977 wurde für die neue Zeitschrift ein 10-köpfiges Herausgeberteam gebildet, das vornehmlich aus österreichischen und deutschen Medizinern bestand. Diesem gehörten neben Prof. Dr. Dr. H. Wbra der Pathologe Prof. Dr. J.H. Holzner und der Internist und Hämatologe Prof. Dr. A. Stacher (Wien) an. Aus Deutschland beteiligten sich die Internisten Prof. Dr. R. Gross (Köln), Prof. Dr. W. Wilmanns (München), Prof. Dr. Dr. St. Tanneberger (Ost-Berlin) und der Immunologe Prof. Dr. K. Munk (Heidelberg). Ferner arbeiteten Prof. Dr. J.P. Obrecht (Basel), Prof. Dr. S. Eckhardt (Budapest) und Prof. Dr. G.P. Murphy (Buffalo, NY) mit. Den Herausgebern wurde ein 30-köpfiger wissenschaftlicher Beirat zugeordnet, der sich aus 25 deutschen Wissenschaftlern unterschiedlicher Disziplinen zusammensetzte, drei weitere kamen aus der Schweiz und je einer aus Österreich und Jugoslawien [1]. Diese Zusammensetzung des «Editorial Boards» wurde prinzipiell bis einschließlich Band 3 (1980) beibehalten.

Mit dem Band 4 (1981) wurde statt der Herausgeberschaft eine «verantwortliche Schriftleitung» eingerichtet. Diese setzte sich aus S. Eckhardt (Budapest), J.H. Holzner (Wien) und G.A. Nagel (Göttingen) zusammen, wobei letzterer die «federführende Schriftleitung» übernahm und die Zeitschrift damit fortan inhaltlich wesentlich gestaltete. Bis zum Band 12 (1989) war der wissenschaftliche Beirat auf 49 Personen angewachsen. In diesem Jahr wurde ich vom Vorstand der Arbeitsgemeinschaft Internistische Onkologie (AIO) der Deutschen

\begin{tabular}{ll}
\hline KARGER & @ 2002 S. Karger GmbH, Freiburg \\
$\begin{array}{l}\text { Fax +497614520714 } \\
\begin{array}{l}\text { E-mail Information@Karger.de } \\
\text { www.karger.com }\end{array}\end{array}$ & Accessible online at: \\
www.karger.com/journals/onk
\end{tabular}

Krebsgesellschaft als Nachfolger für G.A. Nagel vorgeschlagen und übernahm im Sommer 1989 die Schriftleitung. 1990 schied S. Eckhardt nach langjähriger Tätigkeit aus der Schriftleitung aus; im selben Jahr trat auch J.H. Holzner zurück. An seiner Stelle trat ab Band 15 (1992) Prof. Dr. H. Huber (Wien) in die Schriftleitung ein. Ab Band 21 (1998) wurde Prof. Dr. W. Scheithauer (Wien) als dritter Schriftleiter aufgenommen, ab Band 22 (1999) schied H. Huber als Schriftleiter aus [3]. Seit dem Band 22 (1999) tragen W. Scheithauer und ich gemeinsam die schriftleiterische Verantwortung für die Zeitschrift.

\section{Ursprüngliche Konzeption}

Von den Gründern der Jahre 1976-1978 wurde die Zeitschrift ONKOLOGIE ausdrücklich und bewusst als deutschsprachiges onkologisches Fortbildungsorgan konzipiert. So heißt es im Geleitwort des ersten Bandes von 1978:

«Der explosionsartige Anstieg des medizinischen Wissens in den letzten Jahren und Jahrzehnten hat dazu geführt, dass sich viele Spezialgebiete herauskristallisiert haben, die bereits wieder zur Subspezialisierung führen. Dies erschwert es dem einzelnen immer mehr, sich einen Überblick über sein Fachgebiet zu verschaffen, bzw. verlängert oft die Zeit, die von der theoretischen Erkenntnis bis zur praktischen Anwendung beim Patienten verstreicht ... Da ein wesentlicher Teil der Literatur in speziellen Zeitschriften und außerdem in englischer Sprache erscheint, wird es für den Arzt noch schwerer, sich über alles zu informieren ... Aus diesem Grunde haben sich die Herausgeber entschlossen, als Nachfolgezeitschrift der «Österreichischen Zeitschrift für Onkologie» die ONKOLOGIE herauszugeben. Sie ist als Schwesterzeitschrift der in englischer Sprache erscheinenden «Oncology» konzipiert, das heißt, es sollen einige besonders wichtige Beiträge der einen Zeitschrift, ins Englische oder Deutsche übersetzt, auch in die andere aufgenommen werden. Dabei will die OnKOLOGIE vor allem die für die praktische Medizin notwendigen Kenntnisse und Informationen vermitteln, während «Oncology» mehr der aktuellen theoretischen Forschung dienen soll. Die Herausgeber der ONKOLOGIE sind überzeugt, dass es heute mehr denn je notwendig ist, das bereits erarbeitete theoretische und zum Teil hochspezifische Wissen rasch in die klinische Praxis zu überführen...» 


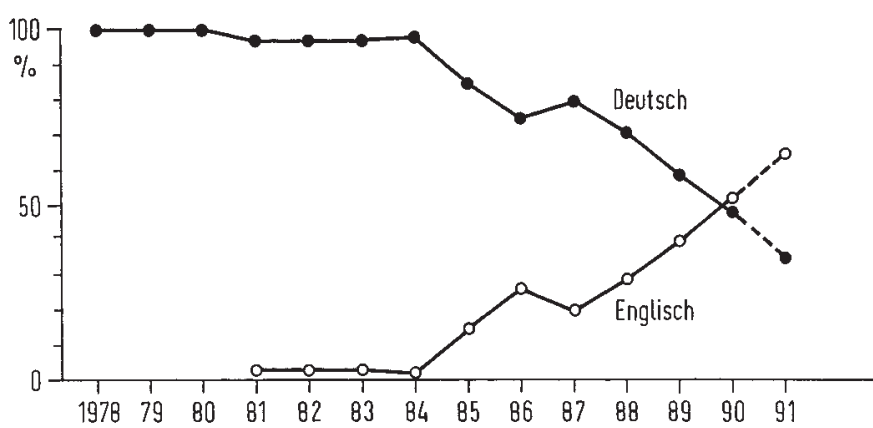

Abb. 1. Anteil der wissenschaftlichen Beiträge in der Zeitschrift ONKOLOGIE pro Jahr in englischer und deutscher Sprache.

Diese Konzeption als onkologisches Fortbildungsorgan ist wohl auch einige Jahre verwirklicht und beibehalten worden, wobei in den Bänden der ersten 10 Jahre keine Arbeiten auszumachen sind, bei denen es sich um Übersetzungen von englischsprachigen Originalarbeiten der Zeitschrift Oncology ins Deutsche handelt.

G.A. Nagel verfolgte indessen in seiner Eigenschaft als Schriftleiter ab 1981 einen anderen Weg, indem er der Zeitschrift ONKOLOGIE ein eigenständiges wissenschaftliches Profil gab. Das wird am Anteil der wissenschaftlichen Beiträge deutlich, die in deutscher bzw. englischer Sprache publiziert worden sind (Abb. 1): In den Jahren 1978-1980 waren die Beiträge zunächst zu 100\% deutschsprachig. Von 1981 an erschien ein kleiner Teil (unter 10\%) in englischer Sprache. Dieser stieg ab 1984 kontinuierlich an und überschritt 1990 die 50\%-Marke. Bei der Übernahme der Schriftleitung im Jahre 1989 ging es uns darum, diese Tendenz für den weiteren Konzeptausbau zu nutzen.

Auch hinsichtlich der Beteiligung verschiedener Fachbereiche waren Veränderungen feststellbar. Die internistische Onkologie/Hämatologie war schon 1978 mit knapp 40\% aller Beiträge führend. Der Anteil stieg bis 1990 weiter auf 65\% an. Dieser Trend war vor allem durch die zunehmende Zahl von sogenannten Therapiestudien bedingt. Der zunehmende Anteil der Beiträge aus der internistischen Onkologie sowie der englischsprachigen Publikationen waren beim weiteren Konzeptausbau der Zeitschrift zu berücksichtigen.

\section{Konzeptausbau seit 1990}

Ab 1990 legte die neue Schriftleitung eine Reihe von Vorschlägen zum weiteren Ausbau der Zeitschrift ONKOLOGIE vor und beschloss in Abstimmung mit dem S. Karger Verlag folgende Änderungen:

Ab 1991 (Band 14) erhielt die OnKOLOGIE den Untertitel «Internationale Zeitschrift für Krebsforschung und -behandlung», ab 1994 (Band 17) die entsprechende englische Bezeichnung «International Journal for Cancer Research and Treatment». Inzwischen hatte sich die Zeitschrift zu einem wichtigen Journal der klinisch orientierten Onkologie entwickelt.
Seit 1992 (Band 15) erscheinen alle Übersichten und wissenschaftlichen Originalarbeiten in englischer Sprache, versehen mit Titel, Kurztitel und Zusammenfassung in Englisch und Deutsch. Seitdem ist auch eine strukturierte Zusammenfassung vorgeschrieben.

Auch Schriftleitung und Beirat wurden mehrfach umstrukturiert: Im Jahr 1991 (Band 14) wurden zusätzlich zu den Schriftleitern sogenannte Fachschriftleiter eingeführt, die ihren jeweiligen Fachbereich in der Zeitschrift vertreten sollten. Ihnen wurden die entsprechenden wissenschaftlichen Beiräte zugeordnet. Die ersten Fachschriftleitungen wurden für die klinisch-experimentelle Onkologie, Radioonkologie, urologische, gynäkologische und chirurgische Onkologie eingerichtet. Ab 1995 (Band 18) kamen die Fachschriftleitung für kasuistische Beiträge, ab 1997 (Band 20) die für onkologische Fortbildung, ab 1998 (Band 21) die für Kommentare und ab 1999 (Band 22) die für internistische Onkologie hinzu.

Von 1997 (Band 20) an wurde der wissenschaftliche Beirat der Zeitschrift strukturiert. Neben den Fächern, die bereits durch einen Fachschriftleiter vertreten wurden, waren im Beirat inzwischen auch die pädiatrische Onkologie, die Dermatoonkologie, die HNO-Onkologie, die Psychoonkologie, die Pathologie, die Biometrie und die onkologische Rehabilitation vertreten. 1998 wurde die Neurochirurgie von der Chirurgie abgetrennt, 1999 kamen die Mund-Kiefer-Gesichts-Chirurgie und die diagnostische Radiologie in der Onkologie hinzu. 2001 (Band 24) umfasste der gesamte wissenschaftliche Beirat 48 Personen, zusammen mit Schriftleitung und Fachschriftleitung besteht das Team zur Zeit aus 58 Personen.

\section{Inhaltliche Ausrichtung}

Die OnKOLOGIE versteht sich als klinisch orientierte onkologisch-hämatologische Fachzeitschrift im mitteleuropäischen Raum. Eine wissenschaftliche Zeitschrift wie die ONKOLOGIE lebt von der Zahl und Qualität der ihr angebotenen Manuskripte. Insofern ist sie im Gegensatz zu jenen Zeitschriften, die sich die Fort- und Weiterbildung zum Ziel gesetzt haben, nicht plan- und steuerbar. Sie ist abhängig von dem wissenschaftlichen «Output» der Fachdisziplin, die sie vertritt. Darüber hinaus bemüht sich die Schriftleitung, zusätzlich thematische Schwerpunkte zu setzen. Solche Schwerpunktthemen waren z.B.:

1. Die frühe klinische Therapieforschung. In diesem $\mathrm{Zu}$ sammenhang wurden Berichte über die Ausstattung von klinischen Einrichtungen für Phase-I- und Phase-II-Prüfungen (1992), die statistischen Erfordernisse der Phase-I (1990), die Implementierung der GCP-Empfehlungen in Deutschland (1994), die Standard Operating Procedures (SOPs) der Phase-I/II-Gruppe der AIO (1994), Hinweise zu klinischen Studien der somatischen Gentherapie (1995), zu den allgemeinen Problemen der Gentherapie $(1997,1999)$ und der Gentechnologie (1998) publiziert. Kürzlich wurde das neue 
Studienhaus der Deutschen Krebsgesellschaft und die neue «Central European Society for Anticancer-Drug Research (CESAR)» mit Sitz in Wien, der sich die deutsche PhaseI/II-Gruppe anschloss, vorgestellt (2000).

2. Neue Substanzen. Im letzten Jahrzehnt wurde in der Zeitschrift ONKOLOGIE über zahlreiche neue Substanzen berichtet, oft noch bevor sie in der klinischen Routine Eingang fanden. So seien beispielhaft Fotemustin, die Etherlipide bzw. Miltefosin (1990, 1992), Vinorelbine (1991) und Droloxifen (1992) genannt. Mehrfach wurden Übersichten über «Neue Wirkstoffe in der Onkologie» publiziert $(1992,1994)$. In jüngerer Zeit erschienen Arbeiten über Pentostatin (1994), Carboplatin (1995), über bispezifische Antikörper und Lobaplatin (1996), Ondensatron (1997), Treosulfan, neue Aromatasehemmer und Capecitabine (1998). Kürzlich wurden Übersichten oder Originalmitteilungen über Rituximab und Ibandronat (1999), Methotrexat-Albumin (MTXHSA) und Natriumfolinat veröffentlicht.

3. Das Dilemma der klinischen Forschung in Deutschland. Ausgehend von einem Grundsatzreferat zu diesem Thema aus der Sicht der internistischen Onkologie (1996), schlossen sich mehrere Stellungnahmen an: aus der Grundlagenforschung (1997), der chirurgischen Onkologie, der forschenden Pharmaindustrie und der Biometrie (1997). Die Serie wurde mit einem Kommentar zur Situation der biomedizinischen Forschung in Österreich abgeschlossen (1998).

4. Meilensteine in Diagnostik und Therapie. Unter diesem Rahmenthema sollten die wesentlichen Entwicklungen eines jeden Fachbereichs mit onkologischem Schwerpunkt geschildert werden. Die Beiträge wurden in lockerer Folge publiziert. Den Anfang machte der Beitrag «Entwicklungen in der Chemotherapie maligner Neoplasien», es folgte «30 Jahre internistische Onkologie in Deutschland» und die Stellungnahme der urologischen und gynäkologischen Onkologie (2000). Diese Serie ist derzeit noch nicht abgeschlossen.

5. Neue Organisationsformen der Onkologie. Die neue Klinik für Tumorbiologie in Freiburg (1991) und das Ergebnis eines Tumornachsorgemodells Nordbaden (1992) wurden vorgestellt. Wir berichteten über den Krebsinformationsdienst (KID) am Deutschen Krebsforschungsinstitut Heidelberg (1993) ebenso wie über eine neue Onkologievereinbarung für niedergelassene Onkologen (1994), über das Modell der onkologischen Brückenpflege (1996) und anlässlich ihres 20-jährigen Bestehens über die Frauenselbsthilfe nach Krebs (1997). Im Jahr ihres 10-jährigen Jubiläums wurde die Phase-I/II-Studiengruppe der AIO vorgestellt (1994). In letzter Zeit publizierten wir eine Stellungnahme zum Onkologischen Zweitmeinungskonsil (Second Opinion) und eine Beschreibung des neuen «Zentrums für Tumordiagnostik und Prävention» in St. Gallen (1999). Kürzlich wurde eine «Praxis für Präventive Onkologie» in Mannheim vorgestellt (2001).
6. Konsensusberichte. Die Schriftleitung der Zeitschrift ONKOLOGIE machte es zu ihrer selbstverständlichen Pflicht, die von entsprechenden Autoritäten und Fachkennern formulierten Konsensusberichte zu publizieren. Solche Berichte liegen zu den kolorektalen Karzinomen (1994; aktualisiert 1999), zum Magenkarzinom (1995), den primären Magenlymphomen (1996), zum Mammakarzinom wie auch zur chronischen lymphatischen Leukämie, zur Haarzelleukämie (1997) und zu den Hodentumoren (1997, 2000) vor.

7. Übersichten zu einzelnen Krebskrankheiten. Die ständig wachsende Information über Pathogenese, Diagnostik und Therapie einzelner onkologischer Krankheitsbilder macht es erforderlich, dieses Wissen für den Leser immer wieder übersichtlich zusammenzustellen. Allein aus den letzten 3 Jahren sind beispielhaft Übersichten über das primäre Magenlymphom, die Chemotherapie des Ösophaguskarzinoms, des Ovarialkarzinoms und des metastasieren Nierenzellkarzinoms (1999), das «Low risk»-MDS, das MDS im Kindesalter, die neoadjuvante und adjuvante Therapie des Magenkarzioms, das nichtkleinzellige Bronchialkarzinom und das multiple Melanom des älteren Menschen zu nennen.

\section{Bibliographische Dienste}

Die Zeitschrift ONKOLOGIE wird in einer großen Zahl von bibliographischen Diensten gelistet, darunter Current Contents, Excerpta Medica, Science Citation Index und seit 2001 auch wieder Index Medicus/Medline. Der Impact Factor von ONKOLOGIE lag für den Zeitraum 1999/2000 (noch vor Wiederaufnahme in Medline) bei 0,640.

\section{Manuskriptaufkommen}

Die Zahl der aus verschiedenen onkologischen Disziplinen pro Jahr publizierten wissenschaftlichen Arbeiten von 1990 bis 2001 ist in Tabelle 1 zusammengestellt. Wie daraus zu ersehen ist, machen die Arbeiten der internistischen Onkologie über einem Zeitraum von 12 Jahren knapp die Hälfte (48\%) aller Publikationen aus. Ausgehend vom Jahre 1990 (65\%) ist dieser Anteil bis 2001 (34\%) kontinuierlich zurückgegangen. Somit werden in den letzten Jahren zwei Drittel aller Beiträge von nicht-internistischen Autoren geschrieben. Das ist eine bewusste Entwicklung, denn es kam der Schriftleitung darauf an, die ONKOLOGIE zu einer interdisziplinären Zeitschrift auszubauen.

Die weiteren klinisch-onkologischen Fächer (Chirurgie, Gynäkologie, Urologie, Pädiatrie, Dermatologie und HNO) sind mit insgesamt $27 \%$, d.h. rund einem Viertel des Gesamtumfangs an der Gestaltung der ONKOLOGIE beteiligt, wobei mit Ausnahme der Urologie in den letzten 12 Jahren kein ansteigender Trend verzeichnet werden konnte. 
Tab. 1. Zahl der in der Zeitschrift ONKOLOGIE aus verschiedenen Fachbereichen publizierten wissenschaftlichen Arbeiten (Reviews, Original Articles, Short Communications, Casuistic Contributions) pro Jahr

\begin{tabular}{|c|c|c|c|c|c|c|c|c|c|c|c|c|c|}
\hline & 1990 & 1991 & 1992 & 1993 & 1994 & 1995 & 1996 & 1997 & 1998 & 1999 & 2000 & 2001 & $\begin{array}{l}\text { gesamt } \\
\mathrm{n}(\%)\end{array}$ \\
\hline Internistische Onkologie & 45 & 30 & 33 & 29 & 29 & 36 & 28 & 20 & 22 & 26 & 22 & 21 & $341(48)$ \\
\hline Chirurgische Onkologie* & 3 & 12 & 9 & 6 & 6 & 7 & 7 & 4 & 6 & 3 & 3 & 6 & $72(10)$ \\
\hline Gynäkologische Onkologie & 2 & 4 & 3 & 4 & 6 & 3 & 0 & 7 & 3 & 4 & 2 & 3 & $41(6)$ \\
\hline Urologische Onkologie & 0 & 2 & 1 & 5 & 3 & 4 & 6 & 3 & 1 & 4 & 5 & 7 & $41(6)$ \\
\hline Pädiatrische Onkologie & 2 & 0 & 0 & 0 & 1 & 1 & 2 & 1 & 1 & 0 & 5 & 0 & $13(2)$ \\
\hline Dermatoonkologie & 0 & 2 & 0 & 0 & 0 & 1 & 1 & 1 & 4 & 1 & 0 & 0 & $10(1)$ \\
\hline HNO-Onkologie & 1 & 2 & 1 & 1 & 0 & 0 & 1 & 1 & 0 & 1 & 3 & 5 & $16(2)$ \\
\hline Radioonkologie & 3 & 4 & 4 & 5 & 2 & 0 & 4 & 3 & 7 & 5 & 6 & 9 & $52(8)$ \\
\hline Psychoonkologie & 0 & 2 & 0 & 0 & 2 & 1 & 2 & 3 & 2 & 0 & 3 & 2 & 17 ( 3$)$ \\
\hline $\begin{array}{l}\text { Klinisch-experimentelle } \\
\text { Onkologie }\end{array}$ & 11 & 5 & 5 & 3 & 14 & 5 & 7 & 7 & 6 & 6 & 9 & 8 & $86(12)$ \\
\hline Biometrie & 2 & 1 & 0 & 0 & 0 & 0 & 0 & 3 & 0 & 0 & 1 & 0 & $7(1)$ \\
\hline Diagnostische Radiologie & - & - & - & - & - & - & 1 & 2 & 0 & 3 & 0 & 0 & $8(1)$ \\
\hline Gesamt & 69 & 64 & 56 & 54 & 63 & 58 & 59 & 55 & 52 & 53 & 60 & 61 & $704(100)$ \\
\hline
\end{tabular}

*einschließlich Neurochirurgie und Orthopädie

Den zweitgrößten Anteil eines Einzelfaches nach der inneren Medizin hat die klinisch-experimentelle Onkologie (12\%). Hierin sind zum einen sehr gute Übersichten, zum anderen aber auch Originalarbeiten aus der experimentellen Medizin enthalten.

\section{Schlussfolgerungen}

Die Zeitschrift OnKOLOGIE wurde von ihren Gründern zunächst als reines Fortbildungsorgan konzipiert. Nach 1990 ist es der Schriftleitung gelungen, das wissenschaftliche Profil kontinuierlich zu steigern. Dieses gelang durch erhebliche Verbesserungen der Organisationsstruktur, Ausbau der Multidisziplinarität, strikte Anwendung des «Peer review»-Systems bei der Auswahl der Manuskripte und nicht zuletzt durch die große Zahl von Autoren, die der ONKOLOGIE ihre exzellenten
Arbeiten zur Publizierung anboten. Seit 2001 wird die ONKOLOGIE auch wieder in Medline geführt.

Die ONKOLOGIE versteht sich heute als führende klinisch-onkologisch orientierte wissenschaftliche Zeitschrift im mitteleuropäischen Raum unter Einbeziehung von Deutschland, Österreich und der Nordschweiz. Erste Anzeichen weisen auf eine gesteigerte Resonanz auch in den osteuropäischen Länder hin. Eine Zeitschrift wie die unsrige kann sich allerdings nicht «aus sich selbst heraus» verbessern, sie ist vielmehr abhängig von der Qualität der Forschung, die in ihrem Einzugsbereich betrieben wird. Für die nahe Zukunft muss es unser Ziel sein, mehr und mehr die besten Arbeiten zur Publizierung in der Zeitschrift ONKOLOGIE zu akquirieren.

\section{Literatur}

1 Impressum. Onkologie 1978;1. 